\title{
Overexpression of Microtubule-Associated Protein-1 Light Chain 3 Is Associated with Melanoma Metastasis and Vasculogenic Mimicry
}

\author{
Chunrong Han, ${ }^{1,3}$ Baocun Sun, ${ }^{1,2}$ Wei Wang, ${ }^{1,4}$ Wenjuan Cai, ${ }^{1,4}$ Dan Lou, ${ }^{2}$ Yan Sun ${ }^{1}$ \\ and Xiulan Zhao ${ }^{2}$ \\ ${ }^{1}$ Department of Pathology, Tianjin Cancer Hospital and Institute, Tianjin Medical University, Tianjin, P.R. China \\ ${ }^{2}$ Department of Pathology, Tianjin Medical University, Tianjin, P.R. China \\ ${ }^{3}$ Department of Pathology, Yangzhou Hongquan Hospital, Jiangsu, P.R. China \\ ${ }^{4}$ Tianjin First Center Hospital, Tianjin, P.R. China
}

\begin{abstract}
Vasculogenic mimicry (VM) is an alternative type of blood supplement and is responsible for aggressive tumor biology and increased tumor-related mortality. Tumor cells obtain oxygen and nutriment through VM channels in the early, rapid-growth stage when blood vessels are insufficient. VM channels are characterized by tubular structures with tumor cells. Autophagy is a catabolic process, by which the cell digests damaged components or organelles of its own cytoplasm in response to nutrient deprivation, hypoxia, and the presence of non-functional protein aggregates. In fact, autophagy plays an important role in normal cell growth, development, and homeostasis. However, it is still controversial whether autophagy is also involved in cell death or cell survival in malignancy. In the present study, we therefore investigated the expression levels of two autophagy-related proteins, microtubule-associated protein-1 light chain 3 (LC3) and beclin-1, with respect to melanoma metastasis and vasculogenic mimicry. Melanoma is characterized by rapid growth, high-metastasis rate, and unpredictable behavior. A total of 70 human melanoma tissues were analyzed, showing that VM was present in 31 melanoma specimens (44.3\%). Melanoma cells displayed high levels of autophagy when VM was present. Real-time quantitative PCR and immunohistochemical analyses showed that the expression levels of beclin-1 and LC3 mRNAs and proteins were both higher in the VM-positive melanoma than those in the VM-negative melanoma $(p<0.05)$. Moreover, the expression of LC3, rather than beclin-1, was strongly associated with metastasis and poor clinical prognosis of human melanoma. Therefore, the enhanced autophagic activity may be related to VM and metastasis of melanoma.
\end{abstract}

Keywords: melanoma; vasculogenic mimicry; microtubule-associated protein 1 light chain 3; beclin-1; melanoma metastasis

Tohoku J. Exp. Med., 2011, 223 (4), 243-251. C 2011 Tohoku University Medical Press

Melanoma is characterized by poorly differentiated rapid growth, high-metastasis rate, and unpredictable behavior (McKinnon et al. 2003). It continues to be one of the most aggressive and fatal malignancies once metastasized. When distant metastasis occurs, the cancer is generally considered incurable. The five-year survival rate is less than $10 \%$, whereas the median survival is $6-12$ months (Balch et al. 2001). Accordingly, inhibiting metastasis can be an effective therapeutic strategy and benefit patients with melanoma. Despite many intensive laboratories and clinical researches, the mechanism involved in metastasis of melanoma still remains unclear.

Some studies have confirmed that the presence of vasculogenic mimicry (VM) channels, a matrix-rich vascularlike network, and the development of fluid-conducting pathways by highly invasive and genetically dysregulated tumor cells are associated with more aggressive and metastatic tumor biology (Maniotis et al. 1999; Hess et al. 2001). VM has been described in highly metastatic malignant tumors, including melanoma (Folberg and Maniotis 2004), inflammatory breast cancer, prostate cancer, hepatocellular carcinoma, ovarian cancer (Foss et al. 1997; Bissell 1999; Hendrix et al. 2003; Sun et al. 2006), synovial sarcoma, rhabdomyosarcoma (Sun et al. 2004), and osteosarcoma (Cai et al. 2004). These findings indicate that VM may facilitate the transmission of tumor cells in the process of metastasis aside from adjunctively supplying blood and nutrients. Many unresolved problems on VM formation in melanoma remain, because most current available investigations on VM are still morphological delineation.

Received December 20, 2010; revision accepted for publication March 2, 2011. doi: 10.1620/tjem.223.243

Correspondence: Baocun Sun, Department of Pathology, Tianjin Medical University, Tianjin, 300070 P.R. China.

e-mail: baocunsun@yeah.net 
Therefore, further investigations have to be conducted to reveal the mechanism involved in VM.

Lazova et al. (2010) investigated 12 cases of cutaneous melanoma of the superficial spreading type, and found that melanoma cells displayed high levels of autophagy phenotype, and the authors suggested that autophagy in melanoma cells might be important to their survival and invasion (Lazova et al. 2010). In cell biology, autophagy is a catabolic process involving the degradation of cell's own components through lysosomal machinery. The proteins encoded by autophagy-related genes (ATGs) are required for the formation of autophagic vesicles (Suzuki and Ohsumi 2007; Xie and Klionsky 2007). Beclin-1, encoded by the gene that is located on human chromosome 17q21, acts in cooperation with the PI3-kinase-signaling complex to enhance the formation of the autophagic vacuole (Liang et al. 1999; Tolkovsky et al. 2002). Yeast ATG8 and its mammalian homologue microtubule-associated protein-1 light chain 3 (LC3) are conjugated to phosphatidylethanolamine in a similar manner with ubiquitination (Ohsumi 2001) and are localized to isolated membranes, thereby playing crucial roles in the formation of autophagosomes (Nakatogawa et al. 2007). LC3 is now widely used as a key molecule to monitor autophagosome formation and autophagy activity in mammalian systems.

Numerous studies on the role of autophagy are currently available (Edinger and Thompson 2003; Levine 2005). Autophagy is generally accepted as a mechanism of cell survival in the presence of genomic injury, endoplasmic reticulum stress, oxidant stress, viral/bacterial infection, nutrient insufficiency, and hypoxia. An autophagy process can serve to regulate the normal turnover of organelles and to remove organelles with compromised functions to maintain homeostasis. However, accumulating data also provide evidence that autophagy can be a backup of the programmed cell death mechanism. There are current controversies on the possible role of autophagy in the process of oncogenesis and tumor progression by promoting cell survival. Some investigators favor the idea that autophagy suppresses tumor development, whereas other investigators support that autophagy enhances tumor development and protects tumor cells from cell death stimuli (Folberg et al. 2000; Kondo et al. 2005; Marx 2006; Wona et al. 2010).

Due to these controversies, investigating the correlation between autophagy and the formation of VM as well as the correlation between autophagy and metastasis in melanoma is of great interest. In this study, we examined the expression of LC 3 and beclin- 1 in 70 cases of human melanoma and determined the role and significance of autophagy, VM channels, and metastasis in melanoma.

\section{Materials and Methods}

\section{Patients}

This study utilized 70 primary tumor specimens of human melanoma obtained from patients who consecutively underwent surgical resection in the Tianjin Medical University Cancer Institute and
Hospital in China between January 1999 and October 2005. This study was conducted in accordance with the declaration of Helsinki. This study was conducted with approval from the Ethics Committee of Tianjin Medical University. Written informed consent was obtained from all participants. The patients comprised of 27 women and 43 men with age ranging from 28-81 years (mean: 54.96 years; SD: 12.60 years). During the follow-up for 113.5 months, 12 patients were found to be still alive, whereas 58 had died. The median survival time was 29.5 months in total.

\section{Clinical and histopathological evaluation}

Seventy tissue slides of melanoma were reviewed using the World Health Organization Classification of Tumors Pathology and Genetics of Skin Tumors (Cai et al. 2004). Tissue sections of $5 \mu \mathrm{m}$ were cut from the formalin-fixed, paraffin-embedded representative tissue blocks of melanoma and were stained with hematoxylin and eosin. The quantities and distribution of VM and other features involved in VM were evaluated in all sections. The number of VM per $400 \times$ field was assessed. Clinicopathologic parameters were obtained from the patients' medical records and files kept at the Department of Pathology, including gender, age, tumor location, survival status, survival time, lymph node status, distant metastasis, American Joint Committee on Cancer (AJCC) stage (AJCC staging system), tumor thickness, conventional necrosis, histology subtypes, melanin, cellular phenotype, and nucleolus.

\section{Immunohistochemical staining}

Serial $4-\mu \mathrm{m}$ sections cut from formalin-fixed, paraffin-embedded tumor tissue for immunohistochemical analysis were deparaffinized and hydrated through a series of xylenes and alcohols before immunohistochemical staining using the alkaline phosphatase-streptavidin method for LC3 and beclin-1. Antigen was firstly rehydrated in phosphate-buffered saline (PBS) before retrieval, and then treated by microwave heating in citrate buffer solution ( $\mathrm{pH}$ 6.0) for 5 min. The sections were incubated with rabbit polyclonal anti-LC3 antibody (Novus Biologicals, USA; dilution of 1:50) and rabbit polyclonal anti-beclin-1 antibody (Santa, USA; dilution of 1:50). Following incubation, the sections were rinsed with PBS and then incubated with biotinylated goat anti-mouse or goat anti-rabbit $\operatorname{IgG}$ for $20 \mathrm{~min}$ at $37^{\circ} \mathrm{C}$. Afterwards, they were incubated with $3,3^{\prime}$-diaminobenzidine chromogen for 5-10 $\mathrm{min}$ at room temperature and then washed with distilled water. Finally, the slides were washed for $5 \mathrm{~min}$ in running tap water and then counterstained with Harris' hematoxylin.

\section{Evaluation of immunohistochemical staining}

LC3 and beclin-1 are considered positive by cytoplasm staining. Semi-quantitative expression levels were determined by assessing both the percentage and intensity of the stained tumor cells. The percentage of positive cells was rated per HPF using $200 \times$ magnification as follows: cases with $<1 \%$ positive cells were rated as $0,1-25 \%$ positive cells as 1 point, $26-50 \%$ positive cells as 2 points, and $>51 \%$ positive cells as 3 points. The staining intensity was rated as follows: 1 point for weak intensity, 2 points for moderate intensity, and 3 points for strong intensity. Points for staining intensity and the percentage of positive cells were added, and the specimens were classified into three groups according to their overall score: negative expression for 0-1 point, weak expression for 2-4 points, and strong expression for 5-6 points. The total scores were as follows: 0-1 (negative) and 2-6 (positive). All slides were evaluated independently by 
two investigators without knowledge of the identity of the patient and the clinical outcome.

Immunohistochemical and periodic acid-Schiff (PAS) histochemical double-staining

After immunohistochemical staining for mouse anti-CD31 monoclonal antibody (Sigma, USA; dilution of 1:200), the sections were exposed to $1 \%$ sodium periodate for $10 \mathrm{~min}$. The sections were then rinsed with distilled water for $5 \mathrm{~min}$, followed by incubating with PAS for $15 \mathrm{~min}$. All the sections were counterstained with hematoxylin, dehydrated, and mounted. Normal human stomach mucous membrane was used as the positive control.

\section{Total RNA isolation and real-time PCR}

Primers were designed and synthesized by TaKaRa Biotechnology Co. Ltd. (Otsu, Shiga, Japan). Real-time PCR analysis was carried out to determine the mRNA expression of LC3 and beclin-1 using the Gene AMP PCR System 7500 Sequence Detector. A separate PCR assay for the mRNA of the $\beta$-actin gene was performed to verify general mRNA integrity. Total RNA was extracted from 30 melanoma tumor samples at $-80^{\circ} \mathrm{C}$ using Trizol reagent. The RNA was then made into cDNA using Taqman reverse transcriptase reagents (Applied Biosystems). The cDNA was used as the template to be amplified in a $25 \mathrm{ml}$ reaction mixture using the following conditions: denaturation at $94^{\circ} \mathrm{C}$ for $5 \mathrm{~min}$ and 35 cycles of $94^{\circ} \mathrm{C}$ for $30 \mathrm{~s}$, the optimal annealing temperature for $45 \mathrm{~s}$, and $55^{\circ} \mathrm{C}$ for $40 \mathrm{~s}$. The primer sequences used for the matrix metalloproteinase LC3 (Accession No.: AF087871.1) detection were 5'-GGCGCTTACAGC TCAATGCTAAT-3' (sense) and 5'-AATTTCATCCCGAAC GTCTCCTG-3' (antisense). The primer sequences used for beclin-1 (Accession no.: NM 003766.3) detection were 5'-GACAGTTTG GCACAATCAATAAC-3' (sense) and 5'-TTCCGTAAGGA ACAAGTCGGTAT-3' (antisense). The primers used to amplify $\beta$-actin were 5'-ATCCGTAAAGACCTCTATGCCAAC-3' (sense) and 5'-ATGGAGCCACCGATCCACA-3' (antisense). The resulting products of LC3, beclin-1, and $\beta$-actin amplification products were 163 , 165 , and $174 \mathrm{bp}$, respectively. The CT value (i.e., the cycle number at which the fluorescence crosses the threshold) was determined, and the formula $2^{\wedge}$ (delta delta $\mathrm{CT}$ ) was used to determine the relative quantity of the amplified fragment. Every sample was tested in triplicate, and the mean value was used.

\section{Statistical analysis}

Statistical analysis was performed in this study using SPSS 13.0. Pearson's $\chi^{2}$, and bivariate correlations were used to determine the different parameters associated with the distribution of VM. $T$-test was used to analyze the mRNA expression of LC3 and beclin-1. The Kaplan-Meier method and the log-rank test were employed to evaluate survival analysis, where $p$-value less than 0.05 was considered significant.

\section{Results}

Morphological characteristics of and quantization for VM

We performed a preliminary screening for the presence of VM in H\&E-stained slides. VM, which was characterized by the channels, were lined by tumor cells instead of endothelial cells without necrosis, hemorrhage, or inflammatory cells in the environment; RBCs were detectable in the lumens as shown in Fig. 1. According to the results of the morphological observation and the CD31/PAS double staining, VM was validated to exist in melanoma. Under a microscope with a $400 \times$ magnification, VM channels were counted in all sections. Among the 70 specimens of melanoma, VM was detected in 31 specimens (44.3\%), as shown in Table 1.

\section{Correlations between VM and clinical histopathologic parameters}

The presence of VM had the close positive correlation with the following parameters in melanoma: mitotic rate ( $p$ $=0.000)$, histological subtype $(p=0.001)$ and Breslow thickness $(p=0.002)$, ulceration $(p=0.001)$, lymph node metastasis $(p=0.003)$, and distant metastasis $(p=0.003)$. However, it had a negative correlation with melanin $(p=$ $0.002)$ and tumor necrosis $(p=0.017)$. Overall, the presence of VM did not show any association with gender, age, tumor location, AJCC stage, cellular phenotype, and nucleolus $(p>0.05)$. The clinical and histopathological characteristics involved in VM are summarized in Table 1.

\section{Expressions of LC3 and beclin-1 at mRNA and proteins} levels

Immunohistochemical staining was performed for autophagy-related proteins LC3 and beclin-1 to detect autophagy in melanoma, as shown in Fig. 1. The result showed that the expression of beclin-1 and LC3 in the VM-positive group was significantly higher than those in the VM-negative group in melanoma $(p<0.05)$ (Table 2). Immunohistochemistry data showed that the presence of VM, the positive LC3 expression, and the positive beclin-1 expression correlated well with each other by Pearson's correlation analysis (Table 3). The result of real-time PCR showed that the expression levels of beclin-1 and LC3 mRNAs in the VM-positive group were both higher than those in the VM-negative group. The difference was significant at $p=0.000$ and $p=0.02$, respectively (Table 4).

\section{Survival analysis}

Kaplan-Meier and log-test survival analyses showed that the total survival time and the metastasis-free survival time for patients with VM were significantly shorter than those for patients without VM $(p<0.05)$. The average survival time of the cases with VM and without VM was $22.711 \pm 1.661,53.566 \pm 5.586$ months, respectively. The total survival and the metastasis-free survival time of patients with positive LC3 expression were significantly shorter compared with those of patients with negative LC3 expression ( $p=0.012$ and $p=0.010)$, respectively. There was no statistical difference between the total survival time and the metastasis-free survival time of patients with positive beclin-1 expression and those of patients with negative beclin-1 expression ( $p=0.973$ and $p=0.347)$, respectively. 
Table 1. Relationship between VM and disease characteristics melanoma patients $(n=70)$.

\begin{tabular}{|c|c|c|c|c|}
\hline \multirow{2}{*}{ Parameter } & \multirow{2}{*}{ No. of patients } & \multicolumn{2}{|c|}{ VM } & \multirow{2}{*}{$P$ value } \\
\hline & & $n(-)$ & $n(+)$ & \\
\hline All patients & 70 & 39 & 31 & \\
\hline \multicolumn{5}{|l|}{ Gender } \\
\hline Male & 27 & 15 & 12 & 0.983 \\
\hline Female & 43 & 24 & 19 & \\
\hline \multicolumn{5}{|l|}{ Age } \\
\hline$\leq 55$ & 38 & 21 & 17 & 0.934 \\
\hline$>55$ & 32 & 18 & 14 & \\
\hline \multicolumn{5}{|l|}{ Tumor location } \\
\hline Trunk & 43 & 20 & 23 & 0.050 \\
\hline Extremities & 27 & 19 & 8 & \\
\hline \multicolumn{5}{|l|}{ Nucleolus } \\
\hline Absent & 36 & 21 & 15 & 0.650 \\
\hline present & 34 & 18 & 16 & \\
\hline \multicolumn{5}{|l|}{ Mitotic rate } \\
\hline$\leq 6 / 10 \mathrm{HPF}$ & 31 & 24 & 7 & $0.000^{*}$ \\
\hline$>7$ and $<20 / 10 \mathrm{HPF}$ & 24 & 13 & 11 & \\
\hline$\geq 20 / 10 \mathrm{HPF}$ & 15 & 2 & 13 & \\
\hline \multicolumn{5}{|l|}{ Histological subtype } \\
\hline $\mathrm{A}+\mathrm{N}+\mathrm{Un}$ & 58 & 23 & 35 & $0.001 *$ \\
\hline SSM & 12 & 9 & 3 & \\
\hline \multicolumn{5}{|l|}{ AJCC stage } \\
\hline $\mathrm{I}+\mathrm{II}$ & 27 & 19 & 8 & 0.050 \\
\hline III + IV & 43 & 20 & 23 & \\
\hline \multicolumn{5}{|l|}{ LN metastesis } \\
\hline Absent & 35 & 24 & 11 & $0.030^{*}$ \\
\hline Present & 35 & 15 & 20 & \\
\hline \multicolumn{5}{|l|}{ Metastasis } \\
\hline Absent & 49 & 33 & 16 & $0.003 *$ \\
\hline Present & 21 & 6 & 15 & \\
\hline \multicolumn{5}{|l|}{ Melanin } \\
\hline Absent & 33 & 12 & 21 & $0.002 *$ \\
\hline Present & 37 & 27 & 10 & \\
\hline \multicolumn{5}{|l|}{ Ulceration } \\
\hline Absent & 49 & 37 & 12 & $0.000^{*}$ \\
\hline Present & 21 & 2 & 19 & \\
\hline \multicolumn{5}{|l|}{ Tumor necrosis } \\
\hline Absent & 34 & 14 & 20 & $0.017 *$ \\
\hline Present & 36 & 25 & 11 & \\
\hline \multicolumn{5}{|l|}{ Celluar phanotype } \\
\hline Spindle & & 14 & 14 & 0.336 \\
\hline Epithelium & & 18 & 9 & \\
\hline Diphase & & 7 & 8 & \\
\hline \multicolumn{5}{|l|}{ Breslow thickness } \\
\hline$\leq 1.5 \mathrm{~mm}$ & 17 & 15 & 2 & $0.002 *$ \\
\hline$\geq 4 \mathrm{~mm}$ & 53 & 24 & 29 & \\
\hline
\end{tabular}

HPF, High-power field; A, Acral lentiginous melanoma (ALM); NM, Nodular melanoma (NM); Un, Untyped melanoma (Un).

$\mathrm{A}+\mathrm{N}+\mathrm{Un}: \mathrm{ALM}+\mathrm{NM}+$ Untyped; ${ }^{*} p<0.05$ 


\section{$\operatorname{VM}(-)$}

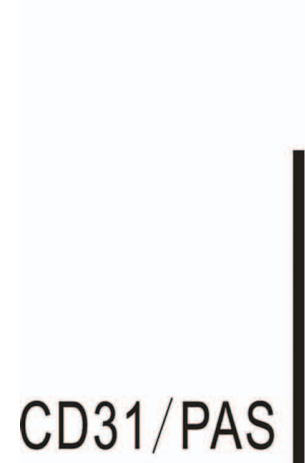

Beclin -1

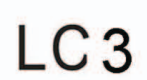

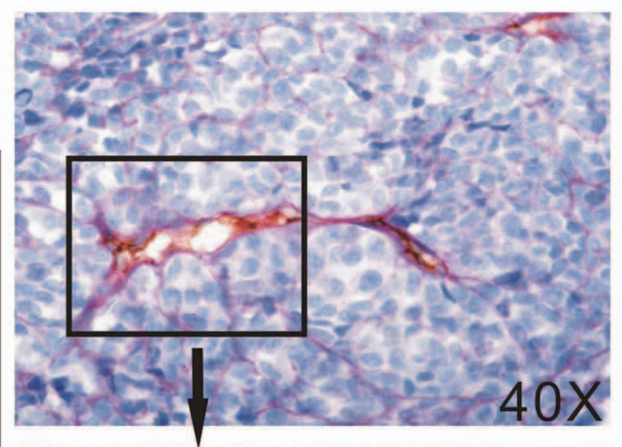
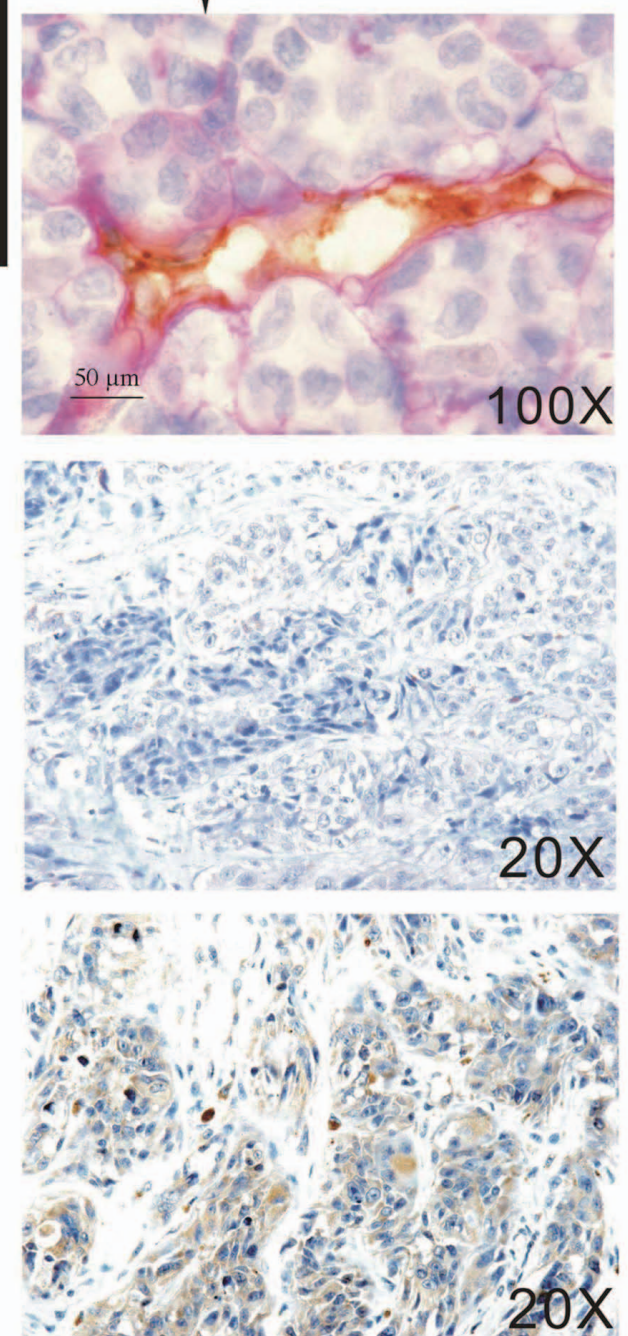

$\operatorname{VM}(+)$
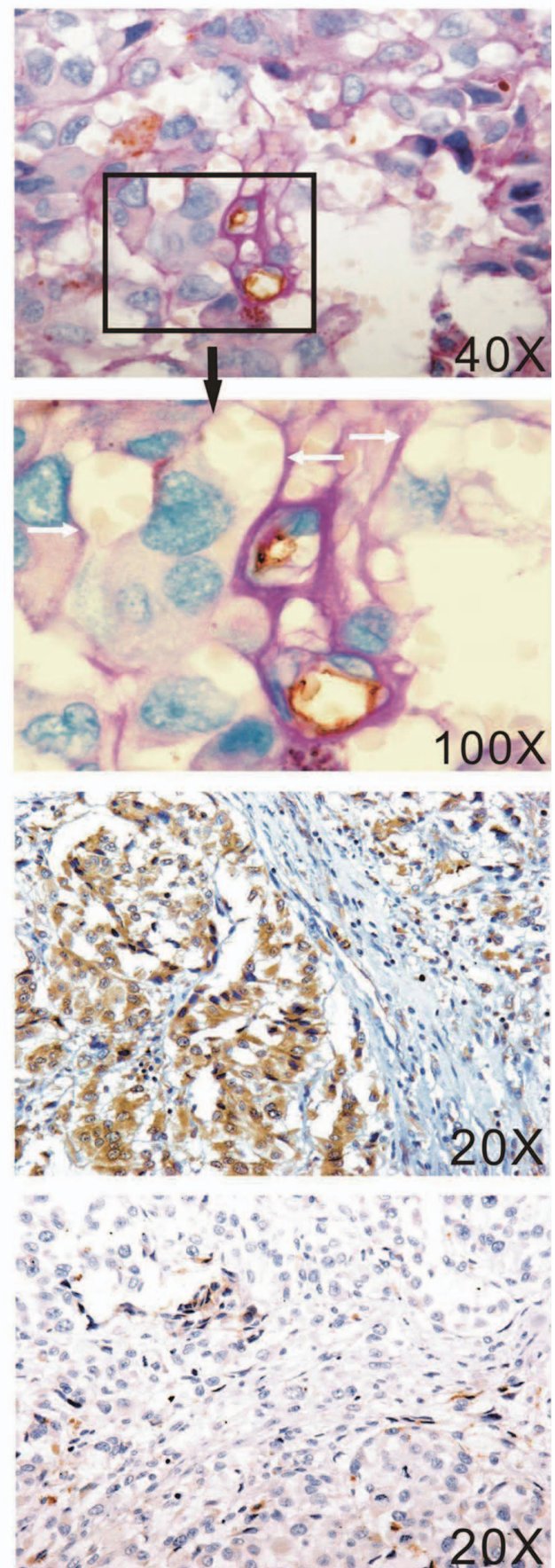

Fig. 1. Immunochemistrical staining of autophagy-related proteins LC3 and Beclin-1 in melanoma.

VM-Positive (right) and VM-Negative (left) was compared by staining of CD31/PAS, LC3 and beclin-1 in human primary melanoma. VM is characterized by the channels, and is lined by tumor cells, instead of endothelial cells. The two pictures of the first line showed the difference of vascular and VM in melanoma by CD31/PAS double staining. The left picture (VM-negative) shows the endothelial cells with CD31/PAS positive, and the right shows that VM with CD31 negative and PAS positive. Scar bar: $50 \mu \mathrm{m}$. The pictures in the second line shows the enlarged part marked with a square in the first line. The third line shows the immunostaining for LC3-negative within the group of VM-negative (left) and LC3-positive within the group of VM-positive (right). The fourth line shows the immunostaining for beclin1-negative within the group of VM-negative (left) and beclin-1-positive within the group of VM-positive (right). 
Table 2. Expressions of LC3, Beclin-1 and disease characteristics melanoma patients $(n=70)$.

\begin{tabular}{|c|c|c|c|c|c|c|c|}
\hline \multirow{2}{*}{ Parameter } & \multirow{2}{*}{ No. of patients } & \multicolumn{2}{|c|}{ LC3 } & \multirow{2}{*}{$P$ value } & \multicolumn{2}{|c|}{ Beclin-1 } & \multirow{2}{*}{$p$ value } \\
\hline & & $n(-)$ & $n(+)$ & & $n(-)$ & $n(+)$ & \\
\hline All patients & 70 & 38 & 32 & & 31 & 39 & \\
\hline \multicolumn{8}{|l|}{ Gender } \\
\hline Male & 27 & 13 & 14 & 0.414 & 11 & 16 & 0.636 \\
\hline Female & 43 & 25 & 18 & & 20 & 23 & \\
\hline \multicolumn{8}{|l|}{ Age } \\
\hline$\leq 55$ & 38 & 18 & 20 & 0.206 & 16 & 22 & 0.689 \\
\hline$>55$ & 32 & 20 & 12 & & 15 & 17 & \\
\hline \multicolumn{8}{|l|}{ Tumor location } \\
\hline Trunk & 43 & 21 & 22 & 0.248 & 20 & 23 & 0.636 \\
\hline Extremities & 27 & 17 & 10 & & 11 & 16 & \\
\hline \multicolumn{8}{|l|}{ Nucleolus } \\
\hline Absent & 36 & 22 & 14 & 0.238 & 16 & 20 & 0.978 \\
\hline present & 34 & 16 & 18 & & 15 & 19 & \\
\hline \multicolumn{8}{|l|}{ Mitotic rate } \\
\hline$\leq 6 / 10 \mathrm{HPF}$ & 31 & 21 & 10 & $0.032 *$ & 15 & 16 & 0.299 \\
\hline$>7$ and $<20 / 10 \mathrm{HPF}$ & 24 & 13 & 11 & & 12 & 12 & \\
\hline$\geq 20 / 10 \mathrm{HPF}$ & 15 & 4 & 11 & & 4 & 11 & \\
\hline \multicolumn{8}{|l|}{ Histological subtype } \\
\hline $\mathrm{A}+\mathrm{N}+\mathrm{Un}$ & 58 & 28 & 30 & 0.026 & 25 & 33 & 0.662 \\
\hline SSM & 12 & 10 & 2 & & 6 & 6 & \\
\hline \multicolumn{8}{|l|}{ AJCC stage } \\
\hline $\mathrm{I}+\mathrm{II}$ & 27 & 17 & 10 & 0.248 & 12 & 15 & 0.983 \\
\hline III + IV & 43 & 21 & 22 & & 19 & 24 & \\
\hline \multicolumn{8}{|l|}{ LN metastesis } \\
\hline Absent & 34 & 19 & 15 & 0.794 & 14 & 20 & 0.611 \\
\hline Present & 36 & 19 & 17 & & 17 & 19 & \\
\hline \multicolumn{8}{|l|}{ Metastasis } \\
\hline Absent & 50 & 32 & 18 & $0.010^{*}$ & 23 & 27 & 0.648 \\
\hline Present & 20 & 6 & 14 & & 8 & 12 & \\
\hline \multicolumn{8}{|l|}{ Melanin } \\
\hline Absent & 33 & 15 & 18 & 0.161 & 15 & 18 & 0.853 \\
\hline Present & 37 & 23 & 14 & & 16 & 21 & \\
\hline \multicolumn{8}{|l|}{ Ulceration } \\
\hline Absent & 49 & 30 & 19 & 0.075 & 27 & 22 & $0.005 *$ \\
\hline Present & 21 & 8 & 13 & & 4 & 17 & \\
\hline \multicolumn{8}{|l|}{ Tumor necrosis } \\
\hline Absent & 34 & 17 & 17 & 0.484 & 15 & 19 & 0.978 \\
\hline Present & 36 & 21 & 15 & & 16 & 20 & \\
\hline \multicolumn{8}{|l|}{ Celluar phanotype } \\
\hline Spindle & 28 & 13 & 15 & 0.093 & 12 & 16 & 0.862 \\
\hline Epithelium & 27 & 19 & 8 & & 13 & 14 & \\
\hline Diphase & 15 & 6 & 9 & & 6 & 9 & \\
\hline \multicolumn{8}{|l|}{ Breslow thickness } \\
\hline$\leq 1.5 \mathrm{~mm}$ & 17 & 13 & 4 & $0.035^{*}$ & 7 & 10 & 0.767 \\
\hline$\geq 4 \mathrm{~mm}$ & 53 & 25 & 28 & & 24 & 29 & \\
\hline
\end{tabular}

HPF, High-power field; A + N + Un: ALM + NM + Untyped; * $p<0.05$ 


\section{Discussion}

VM has been considered an important mechanism involved in melanoma metastasis (Sun et al. 2006), but the role of autophagy is not well known. In this context, there are similar aspects between autophagy and VM in the growth and progress of tumors. First, morphologically, VM channels are observed under a microscope and are characterized by tubular structures with tumor cells, but not endothelial cells, pasted on the surface of the basement membrane (Maniotis et al. 1999). VM is accepted as a novel blood supply under the tumor microenvironment in several different types of cancers, which are all highly malignant and can easily metastasize (Folberg and Maniotis 2004). Tumor cells can obtain oxygen and nutriment through a VM channel in the early, rapid-growth stage when blood vessels are insufficient. Morphologically, autophagy is described for structures that are observed using electron microscopy and that are characterized by the formation of autophagosomes consisting of single- or double-membrane lysosomalderived vesicles containing cytoplasmic particles, including organelles, in various stages of disintegration. Many observers have argued that autophagy might actually be a survival mechanism benefiting the tumor cells. Second, in the formation mechanism, there are three important elements in VM formation: the property of plasticity and modification of malignant tumor cells, the remodeling of the extracellular matrix, and the connection of the VM channels to the host microcirculation system (Yorimitsu and Klionsky 2005; Zhang et al. 2006). All are regulated by a hypoxic and ischemic microenvironment. Analogically, autophagy is a cellular response to both extracellular (i.e., nutrient deprivation or hypoxia) and intracellular (i.e., accumulation of damaged organelles and cytoplasmic compo-

Table 3. Correlations between VM and immunohistochemical parameters in melanoma.

\begin{tabular}{ccc}
\hline & Beclin-1 $(p$ value $)$ & VM $(p$ value $)$ \\
\hline VM & $0.038^{*}$ & \\
LC3 & $0.012^{*}$ & $0.010^{*}$ \\
\hline
\end{tabular}

$* p<0.05$ nents) stress conditions. During nutrient starvation, autophagy is a major mechanism by which tumor cells increase the breakdown of non-vital components and the reallocation of nutrients, ensuring that more essential processes (Sun et al. 2007). Third, both VM and autophagy are genetically controlled processes that result in accommodating and reforming the microenvironment.

Thus, we can speculate that tumors become more selfsufficient through these two different mechanisms of autophagy and VM to obtain the common aim of surviving and progressing in an unfavorable microenvironment. This hypothesis is verified in our current study. We carefully assessed the distribution of VM in human melanoma. In the H\&E stained sections, we observed that VM channels were composed of a basement membrane with positive PAS staining and CD31-negative reagent in the absence of endothelial cells and fibroblasts. Tumor cells from tubular structures and mimic the pattern of embryonic vasculogenic networks; no hemorrhage, necrosis, or inflammatory cells are found around VM networks. The presence of VM is closely associated with mitotic rate, histological subtype, Breslow thickness, ulceration, lymph node metastasis, and distant metastasis in melanoma. This result is consistent with earlier investigations (Sun et al. 2008; Morselli et al. 2009). In addition, we found that the presence of VM is reversely associated with melanin and tumor necrosis.

In this study, autophagy was measured by microscopic quantitative analysis of cells expressing LC3 and beclin-1, which are two important members of autophagy-related proteins promoted and expressed in the autophagic pathway (Ahn et al. 2007). Immunohistochemistry and real-time PCR results showed that the expression levels of beclin-1 and LC3 in the VM-positive group were significantly higher than those in the VM-negative group in melanoma. Our results can be supported by the first reported that melanoma cells could display high levels of autophagy phenotype (Lazova et al. 2010). In our study, the expression of beclin-1 is consistent with the result in colorectal and gastric cancer (Liang et al. 1999). However, it is contrary to the result in breast carcinoma cells in which the expression of beclin-1 is decreased (Ohsumi 2001). In our study, immunohistochemical staining results showed that the positive LC3 expression was related to mitotic rate, metastasis,

Table 4. The mRNA expression of LC3 and Beclin-1 in melanoma.

\begin{tabular}{ccccc}
\hline Gene & Group & $\bar{x} \pm$ s.D. & $t$ & $p$ value \\
\hline Beclin-1 & & & 6.948 & $0.000^{*}$ \\
& VM + & $6.9378 \pm 2.256$ & & \\
& VM - & $1.260 \pm 0.492$ & & $0.000^{*}$ \\
LC3 & & & \\
& VM + & $5.793 \pm 1.469$ & & \\
& VM - & $2.124 \pm 0.963$ & & \\
\hline
\end{tabular}

* The $p$ value was considered 0.000 , because $p$ values of Beclin- 1 and LC 3 were 0.000121 and 0.000082 , respectively. 
and Breslow thickness, and the positive Beclin-1 expression was only related to ulceration. In addition, the presence of VM, the positive expression of LC3 and Beclin- 1 correlated well with each other by Pearson's correlation analysis. Thus, we can conclude that VM and autophagy are strongly associated with each other under a similar tumor microenvironment.

The Kaplan-meier method and Log-rank test data showed that the survival time of patients with VM positive tumor was shorter than that of those without VM. This is similar to previous study (Zhang et al. 2009). The total survival and metastasis-free survival time of patients with positive LC3 expression were significantly shorter than those of patients with negative LC 3 expression, $(p=0.012$ and $p$ $=0.010)$, respectively. We did not find that the total survival time and the metastasis-free survival time of patients with positive beclin-1 expression were different from those of patients with negative beclin-1 expression ( $p=0.973$ and $p=0.347)$, respectively. Therefore, we concluded that LC3, but not beclin-1, is closely associated with VM, metastasis, and poor clinical prognosis in human melanoma.

Under a microenvironment without adequate oxygen, nutrition, and blood supply, some tumor cells, especially apoptosis-resistant cells such as melanoma cells are likely to be self-sufficiently regulated by some ATGs and VM-related genes. Based on our data, we confirmed that the role of autophagy was beneficial for the bulk of tumor population in its growth, invasion, and metastasis, similar to the role of VM regardless of autophagy-enhanced cell survival or cell death. Certainly, autophagy mechanism in melanoma is still poorly understood. Further study on a larger scale is necessary to explain the role and significance of autophagy and VM expression in melanoma. Recently, autophagy has been demonstrated to be important in conferring resistance to chemotherapy, radiation therapy, and immunotherapy (Livesey et al. 2009). Inappropriate drug delivery, secondary toxicities, and persistent chemo- and immunoresistance have traditionally compromised treatment response in primary and metastatic melanoma. Therefore, we suppose that the treatment strategy of inhibiting LC3 might be effective in metastasis melanoma by depriving cells of an important energy supply source.

\section{Acknowledgments}

This study was supported by the National Natural Science foundation of China (No: 30770828).

\section{Conflict of Interest}

All authors have no conflict of interest regarding this paper.

\section{References}

Ahn, C.H., Jeong, E.G., Lee, J.W., Kim, M.S., Kim, S.H., Kim, S.S., Yoo, N.J. \& Lee, S.H. (2007) Expression of beclin-1, an autophagy-related protein, in gastric and colorectal cancers. APMIS, 115, 1344-1349.
Balch, C., Buzaid, A.C., Soong, S.J., Atkins, M.B., Cascinelli, N., Coit, D.G., Fleming, I.D., Gershenwald, J.E., Houghton, A. Jr., Kirkwood, J.M., McMasters, K.M., Mihm, M.F., Morton, D.L., Reintgen, D.S., Ross, M.I., Sober, A., Thompson, J.A. \& Thompson, J.F. (2001) Final version of the American Joint Committee on Cancer staging system for cutaneous melanoma. J. Clin. Oncol., 19, 3635-3648.

Bissell, M.J. (1999) Tumor plasticity allows vasculogenic mimicry, a novel form of angiogenic switch. A rose by any other name? Am. J. Pathol., 155, 675-679.

Cai, X.S., Jia, Y.W., Mei, J. \& Tang, R.Y. (2004) Tumor blood vessels formation in osteosarcoma: vasculogenesis mimicry. Chin. Med. J. (Engl.), 117, 94-98.

Edinger, A.L. \& Thompson, C.B. (2003) Defective autophagy leads to cancer. Cancer. Cell, 4, 422-424.

Folberg, R., Hendrix, M.J. \& Maniotis, A.J. (2000) Vasculogenic mimicry and tumor angiogenesis. Am. J. Pathol., 156, 361381.

Folberg, R. \& Maniotis, A.J. (2004) Vasculogenic mimicry. APMIS, 112, 508-525.

Foss, A.J., Alexander, R.A., Hungerford, J.L., Hungerford, J.L., Harris, A.L., Cree, I.A. \& Lightman, S. (1997) Reassessment of the PAS patterns in uveal melanoma. Br. J. Ophthalmol., 81, 240-246.

Hendrix, M.J., Seftor, E.A., Hess, A.R. \& Seftor, R.E. (2003) Vasculogenic mimicry and tumour-cell plasticity: lessons from melanoma. Nat. Rev. Cancer, 3, 411-421.

Hess, A.R., Seftor, E.A., Gardner, L.M., Carles-Kinch, K., Schneider, G.B., Seftor, R.E., Kinch, M.S. \& Hendrix, M.J. (2001) Molecular regulation of tumor cell vasculogenic mimicry by tyrosine phosphorylation: role of epithelial cell kinase (Eck/EphA2). Cancer Res., 61, 3250-3255.

Kondo, Y., Kanzawa, T., Sawaya, R. \& Kondo, S. (2005) The role of autophagy in cancer development and response to therapy. Nat. Rev. Cancer, 5, 726-734.

Lazova, R., Klump, V. \& Pawelek, J. (2010) Autophagy in cutaneous malignant melanoma. J. Cutan. Pathol., 37, 256-268.

Levine, B. (2005) Eating oneself and uninvited guests: autophagyrelated pathways in cellular defense. Cell, 120, 159-162.

Liang, X.H., Jackson, S., Seaman, M., Brown, K., Kempkes, B., Hibshoosh, H. \& Levine, B. (1999) Induction of autophagy and inhibition of tumorigenesis by beclin 1. Nature, 402, 672-676.

Livesey, K.M., Tang, D., Zeh, H.J. \& Lotze, M.T. (2009) Autophagy inhibition in combination cancer treatment. Curr. Opin. Investig. Drugs, 10, 1269-1279.

Maniotis, A.J., Folberg, R., Hess, A., Seftor, E.A., Gardner, L.M., Pe'er, J., Trent, J.M., Meltzer, P.S. \& Hendrix, M.J. (1999) Vascular channel formation by human melanoma cells in vivo and in vitro: vasculogenic mimicry. Am. J. Pathol., 155, 739752.

Marx, J. (2006) Autophagy: is it cancer's friend or foe? Science, 312, 1160-1161.

McKinnon, J.G., Yu, X.Q., McCarthy, W.H. \& Thompson, J.F. (2003) Prognosis for patients with thin cutaneous melanoma: long-term survival data from New South Wales Central Cancer Registry and the Sydney Melanoma Unit. Cancer, 98, 12231231.

Morselli, E., Galluzzi, L., Kepp, O., Vicencio, J.M., Criollo, A., Maiuri, M.C. \& Kroemer, G. (2009) Anti- and pro-tumor functions of autophagy. Biochim. Biophys. Acta, 1793, 15241532 .

Nakatogawa, H., Ichimura, Y. \& Ohsumi, Y. (2007) Atg8, a ubiquitinlike protein required for autophagosome formation, mediates membrane tethering and hemifusion. Cell, 130, 165-178.

Ohsumi, Y. (2001) Molecular dissection of autophagy: two ubiquitin-like systems. Nat. Rev. Mol. Cell Biol., 2, 211-216.

Sun, B., Qie, S. Zhang, S., Sun, T., Zhao, X., Gao, S., Ni, C., Wang, X., Liu, Y. \& Zhang, L. (2008) Role and mechanism of 
vasculogenic mimicry in gastrointestinal stromal tumors. Hum. Pathol., 39, 444-451.

Sun, B., Zhang, D., Zhang, S., Zhang, W., Guo, H. \& Zhao, X. (2007) Hypoxia influences vasculogenic mimicry channel formation and tumor invasion-related protein expression in melanoma. Cancer Lett., 249, 188-197.

Sun, B., Zhang, S., Zhang, D., Du, J., Guo, H., Zhao, X., Zhang, W. \& Hao, X. (2006) Vasculogenic mimicry is associated with high tumor grade, invasion and metastasis, and short survival in patients with hepatocellular carcinoma. Oncol. Rep., 16, 693-698.

Sun, B., Zhang, S., Zhao, X., Zhang, W. \& Hao, X. (2004) Vasculogenic mimicry is associated with poor survivalin patients with mesothelial sarcomas and alveolar rhabdomyosarcomas. Int. J. Oncol., 25, 1609-1614.

Suzuki, K. \& Ohsumi, Y. (2007) Molecular machinery of autophagosome formation in yeast Saccharomyces cerevisiae. FEBS Lett., 581, 2156-2161.

Tolkovsky, A.M., Xue, L., Fletcher, G.C. \& Borutaite, V. (2002)
Mitochondrial disappearance from cells: a clue to the role of autophagy in programelanomaed cell death and disease? Biochimie, 84, 233-240.

Wona, K.Y., Kima, G.Y., Kimb, Y.W., Song, J.Y. \& Lim, S.J. (2010) Clinicopathologic correlation of beclin-1 and bcl-2 expression in human breast cancer. Hum. Pathol., 41, 107112.

Xie, Z. \& Klionsky, D.J. (2007) Autophagosome formation: core machinery and adaptations. Nat. Cell Biol., 10, 1102-1109.

Yorimitsu, T. \& Klionsky, D.J. (2005) Autophagy: molecular machinery for self-eating. Cell Death Differ., 12, 1542-1552.

Zhang, S., Guo, H., Zhang, D., Zhang, W., Zhao, X., Ren, Z. \& Sun, B. (2006) Microcirculation patterns in different stages of melanoma growth. Oncol. Rep., 15, 15-20.

Zhang, S.W., Li, M., Zhang, D., Xu, S., Wang, X., Liu, Z., Zhao, X. \& Sun, B. (2009) Hypoxia influences linearly patterned programelanomaed cell necrosis and tumor blood supply patterns formation in melanoma. Lab. Invest., 89, 575-586. 